\title{
Coherent pump-probe interactions and terahertz intersubband gain in semiconductor quantum wells
}

\author{
Ansheng Liu ${ }^{\S}$ and C. Z. Ning \\ NASA Ames Research Center, M/S T27A-1, Moffett Field, CA 94035 \\ ${ }^{\S}$ Department of Electrical Engineering, Arizona State University, Ternpe, AZ 85287
}

Fax: (650)604 395\%; Email: cning@nas.nasa.gov

In recent years there has been considerable interest in intersubband-transition-based infrared semiconductor quantum well (QW) lasers because of their potential applications. In the mid-infrared range, both electrically-injected quantum cascade lasers [1] and opticallypumped multiple QW lasers [2] have been experimentally realized. In these studies, optical gain is due to population inversion between the lasing subbands. It was also proposed that stimulated Raman scattering in QW systems can produce net infrared optical gain [3]. In such a nonlinear optical scheme, the appearance of optical gain that may lead to intersubband Raman lasers does not rely on the population inversion. Since, in the resonant Raman process (Raman gain is the largest in this case), the pump field induces population rolistribution among subbands in the QW system, it seems that a realistic estimate of the optical garin his to include this effect. Perturbative calculations used in the previous work [3] may orerestimate the Raman gain.

In this paper we present a nonperturbative calculation of terahertz gain of opticallypumped semiconductor step quantum wells. Limiting optical transitions within the conduction band of the QW, we solve the pump-field-induced nonequilibrium distribution function for each subband of the QW system from a set of coupled rate equations. Both intrasubband and intersubband relaxation processes in the quantum well system are included. Taking into account the coherent interactions between pump and $\mathrm{THz}$ (signal) waves, we derive the susceptibility of the QW system for the THz field. For a Ga.4s/AlGaAs step QW, we calculate the THz gain spectrum for different pump frequencies and intensities. Under moderately strong pumping $\left(\sim 0.3 \mathrm{MW} / \mathrm{cm}^{2}\right)$, a significant $\mathrm{THz}$ gain $\left(\sim 300 \mathrm{~cm}^{-1}\right)$ is predicted. It is also shown that the coherent wave interactions (resonant stimulated Raman processes) contribute significantly to the $\mathrm{THz}$ gain. Our theory is outlined as follows.

We consider an asymetric step quantum well structure with three subbands. The tro upper subbands are the lasing states, and the subband energy separation $\left(E_{32}\right)$ at zero wave vector of electrons $\left(k_{\mathrm{li}}=0\right)$ lies in the THz frequency range (1-10 THz or $\left.4-40 \mathrm{meV}\right)$. The condiction band nonparabolicity is taken into account by using a subband-energy-dependent effective mass scheme. A strong pump, field of frequency $\omega_{p}\left(h \omega_{p} \approx E_{31}\right)$ drives the QW systenn, as illustrated in the inset of Fig. 1. The pump field induces a redistribution of electrons annong the three subbands. The rate erdiations that govern the electronic distribution functions $\left[\int_{m}\left(k_{i l}\right) \equiv \int_{m}(m=1,2,3)\right]$ are given by $[4]$

$$
\begin{aligned}
& \frac{\partial f_{1}}{\partial l}=-\Pi_{12}\left(f_{1}-f_{2}\right)-\Pi_{13}\left(f_{1}-f_{3}\right)-\frac{f_{1}-f_{1}^{F}}{T_{c r}}+\frac{f_{2}}{\tau_{21}}+\frac{f_{3}}{\tau_{31}},
\end{aligned}
$$

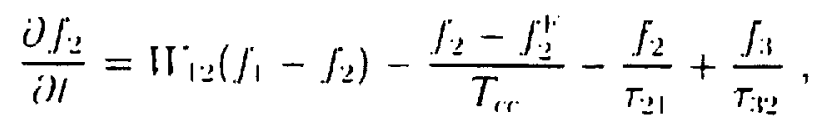




$$
\frac{\partial f_{3}}{\partial t}=W_{13}\left(f_{1}-f_{3}\right)-\frac{f_{3}-f_{3}^{\mathrm{F}}}{T_{c c}}-\frac{f_{3}}{\tau_{31}}-\frac{f_{3}}{\tau_{32}}
$$

where

$$
W_{m n}=\frac{2 \Gamma_{m n}}{\hbar} \frac{\left|H_{m n}^{\prime}\right|^{2}}{\left[\hbar \omega_{p}-E_{n m}\left(k_{\|}\right)\right]^{2}+\Gamma_{m n}^{2}} .
$$

In the above equations $T_{c c}, \tau_{m n}$, and $\Gamma_{m n}$ are the intrasubband carrier-carrier scattering time, intersubband relaxation time, and line broadening, respectively. In the electrical dipole approximation, the matrix element of the light-quantum-well interaction Hamiltonian is $H_{m n}^{\prime}=-E_{p} \mu_{n n n}$, where $E_{p}$ denotes the amplitude of the pump electric field that is polarized perpendicular to the wells and $\mu_{m n}$ is the dipole matrix element between subband $m$ and subband $n$. The quantities $f_{m}^{\mathrm{F}}(m=1,2,3)$ are considered to be the quasi-equilibrium distribution functions since the intrasubband relaxation processes in QW systems are usually very fast (in fs rańges). For a given temperature and electron density, the steady-state nonequilibrium distribution function for each subband is uniquely determined from Eqs. (1)(3). Here we would like to emphasize that the total particle number in our QW system is conserved in our treatment.

Taking into account the pump and $\mathrm{THz}$ wave interactions in the QW system, we find the off-diagonal element of the density matrix operator between subband 3 and subband 2 at the THz frequency $\omega$ is given by [4]

$$
\rho_{32}(\omega)=\frac{f_{2}-f_{3}-\mathcal{R}\left(\omega, \omega_{p}\right)}{\hbar \omega-E_{32}\left(k_{\|}\right)+i \Gamma_{23}-\Delta\left(\omega, \omega_{p}\right)}\left(-\mu_{32} E_{\omega}\right) \equiv-A\left(\omega, \omega_{p}, k_{\|}\right) \mu_{32} E_{\omega}
$$

where $E_{\omega}$ is the THz field in the QW, and

$$
\begin{aligned}
\Delta\left(\omega, \omega_{p}\right)= & \frac{\left|H_{31}^{\prime}\right|^{2}}{\hbar\left(\omega-\omega_{p}\right)+E_{21}\left(k_{\| 1}\right)+i \Gamma_{12}}+\frac{\left|H_{21}^{\prime}\right|^{2}}{\hbar\left(\omega_{p}+\omega\right)-E_{31}\left(k_{\| 1}\right)+i \Gamma_{13}}, \\
\mathcal{R}\left(\omega, \omega_{p}\right)= & \frac{\left|H_{31}^{\prime}\right|^{2}\left(f_{1}-f_{3}\right)}{\left[\hbar\left(\omega-\omega_{p}\right)+E_{21}\left(k_{\| 1}\right)+i \Gamma_{12}\right]\left[\hbar \omega_{p}-E_{31}\left(k_{i \|}\right)-i \Gamma_{13}\right]} \\
& +\frac{z=\left|H_{21}^{\prime}\right|^{2}\left(f_{1}-f_{2}\right)}{\left[\hbar\left(\omega_{p}+\omega\right)-E_{31}\left(k_{\| 1}\right)+i \Gamma_{13}\right]\left[\hbar \omega_{p}-E_{21}\left(k_{11}\right)+i \Gamma_{12}\right]} .
\end{aligned}
$$

$\Delta\left(\omega, \omega_{p}\right)$ in $E_{q} .(5)$ is responsible for the optical Stark effect while $\mathcal{R}\left(\omega, \omega_{p}\right)$ gives rise to the Raman gain of the QW system. From Eq.-(5) we define the susccptibility for the THz probe ficlet as

$$
\chi(\omega)=-\frac{2\left|\mu_{23}\right|^{2}}{\epsilon_{0} L_{\mathrm{w}}} \iint \frac{d^{2} k_{\|\|}}{(2 \pi)^{2}} \mathrm{~A}\left(\omega, \omega_{p}, k_{\|}\right),
$$

$I_{\mathrm{w}}$ being the width of the active layer. Therefore, the optical gain is given by

$$
G(\omega)=-\frac{\omega}{r_{0} n_{b}} \operatorname{Im}[x(\omega)] .
$$

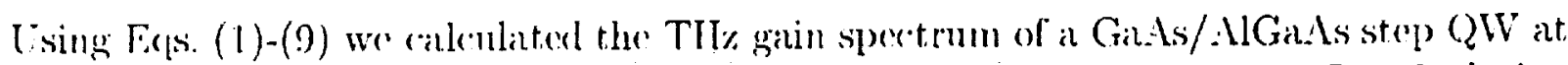

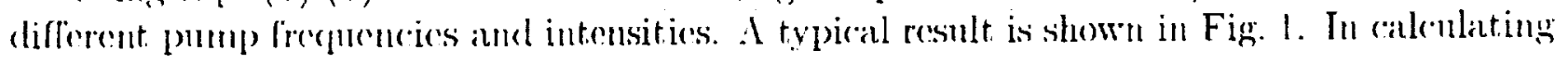




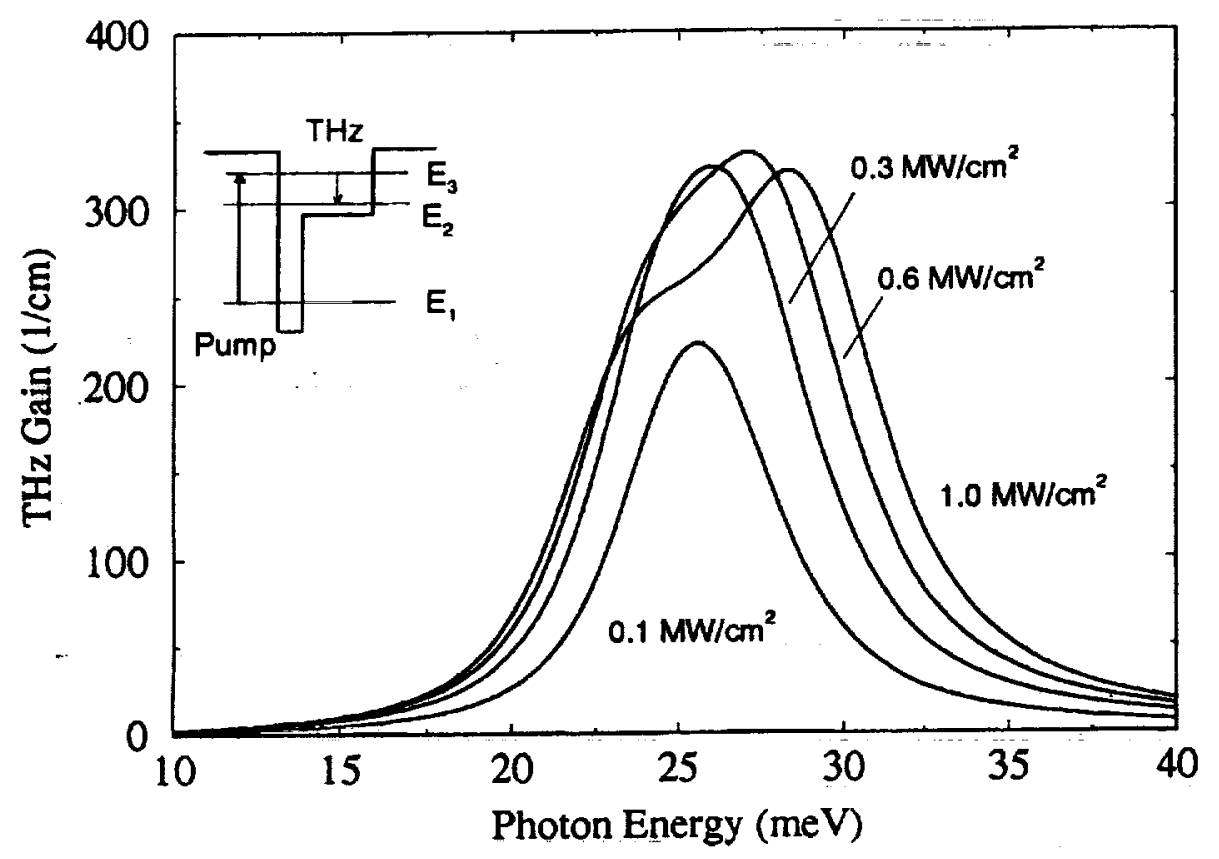

Figure 1: THz gain spectrum of a GaAs/AlGaAs step quantum well with a subband separation of $E_{32} \approx 25 \mathrm{meV}$ for different pump intensities, i.e. $0.1,0.3,0.6$, and $1.0 \mathrm{MW} / \mathrm{cm}^{2}$. The pump photon energy is $\hbar \omega_{p}=E_{31} \approx 125 \mathrm{meV}$. The other parameters used in the calculation are given as follows: the sheet electron density is $5.16 \times 10^{10} \mathrm{~cm}^{-2}, T_{c c}=0.6 \mathrm{ps}, \tau_{21}=1.0 \mathrm{ps}$, $\tau_{31}=1.2 \mathrm{ps}, \tau_{32}=1.5 \mathrm{ps}$, and $\Gamma_{12}=\Gamma_{13}=\Gamma_{23}=3.0 \mathrm{meV}$.

Fig. 1, the following parameters were employed: the deep well width is $65 \AA$, the shallow well width is $130 \AA, \Gamma_{12}=\Gamma_{13}=\Gamma_{23}=3.0 \mathrm{meV}, T_{c c}=0.6 \mathrm{ps}, \tau_{21}=1.0 \mathrm{ps}, \tau_{31}=1.2 \mathrm{ps}$, and $\tau_{32}=1.5 \mathrm{ps}$. The sheet electron density is $5.16 \times 10^{10} \mathrm{~cm}^{-2}$. The temperature is $\mathrm{T}=100 \mathrm{~K}$. The pump photon energy is $\hbar \omega_{p}=E_{31} \approx 125 \mathrm{meV}$. The pump intensity is varied from 0.1 to $1.0 \mathrm{MW} / \mathrm{cm}^{2}$. Our calculations show that the optical gain is strongly dependent on the pump intensity and frequency, and the maximum gain is not linearly proportional to the pump intensity as given in the previous perturbative calculations [3] even when the pump field is moderately strong because of the optical Stark effect as well as the pump-induced population redistribution among the subbands.

1. J. Faist, F. Capasso, D. Sivco, C. Sirtori, A. L. Hutchinson, S. N. G. Chu, and A. Y. Cho, Science 264, 553 (1994); Appl. Phys. Lett. 68, 3680 (1996).

2. O. Gauthier-Lafaye et. al., Appl. Phys. Lett. 71, 3619 (1997).

3. J. B. Khurgin, G. Sun, L. R. Friedmau, and R. A. Soref, J. Appl. Phys. 78, 7398 (1995); .J. Opt. Soc. Am. B 15, 6.48 (1998).

1. A. Lin, J. Opt. Soc. Am. B 15, 1741 (1998). 